\title{
The Effect of Program Genre on Modes of Media Multitasking and Ad Processing
}

\author{
Hyejin Bang ${ }^{1}$ \\ College of Business Administration, Kookmin University, Seoul, Republic of Korea
}

\begin{abstract}
Despite many studies on the effect of MM on advertising, previous studies on $\mathrm{MM}$ considered $\mathrm{MM}$ a homogenous phenomenon. Further, whether and how media-related factors predict different modes of MM and how this behavior affects ad processing has been unknown. To fill this gap, the purpose of this study was to examine (a) the effect of program-genre on the occurrence of different modes of media multitasking (MM; utilitarian MM vs. hedonic MM) and (b) how different modes of MM influence the way viewers process ads on the primary screen (i.e., computer screen). A lab-based experiment yielded data for testing the hypotheses. The findings suggest that findings suggest that MM can be classified into two distinct modes: utilitarian MM and hedonic MM. Further, the findings show that participants who watched the sitcom tended to engage in a higher amount of utilitarian MM than those who watched the suspenseful drama; however, both groups engaged in a comparable amount of hedonic MM. The findings also indicate that participants who watched the sitcom demonstrated a lower level of ad memory than those who watched the suspenseful drama. The current study provides meaningful theoretical implications. Further, this study provides useful implications for advertising practitioners and marketers.

Keywords: Media multitasking, Context effect, Ad processing, Limited Cognitive Capacity
\end{abstract}

\section{Introduction}

Consider the following scenario: Sarah and her brother Mike are sitting together on the couch watching a popular show, Saturday Night Live. As always, both of them are holding their smartphones, and their laptops and iPads are also within reach. While watching the show, Sarah receives a notification from Instagram that her best friend, who is now on vacation in Italy, uploaded new pictures on her feed. To check her status and look at the pictures, Sarah opens the Instagram app on her smartphone and browses the feed while trying to follow the television show. At the same time, Mike suddenly remembers that he has to book a flight to

\footnotetext{
${ }^{1}$ Corresponding author:: hjbang@kookmin.ac.kr
} 
New York for his friend's upcoming wedding. To check for a good deal, he grabs his laptop and searches Expedia and Orbitz while watching the show.

This scenario represents a normal pattern of media consumption: media multitasking (MM), the simultaneous use of two or more media devices at a time [1-3]. Responding to the pervasiveness of this phenomenon, scholars have investigated several questions associated with information processing and media effects related to MM. For instance, prior studies have found that $\mathrm{MM}$ resulted in diminished attention, lower quality of exposure, shallow processing, and decreased memory of messages (e.g., ads) in the media [4-6]. On the other hand, MM might also inhibit counter argumentation and increase enjoyment and acceptance of messages [7].

Upon closer examination, the MM behavior of Sarah and Mike indicates two different types of MM. Sarah's second screening can be categorized as more hedonic or entertaining MM, while Mike's can be regarded as more goal-oriented, utilitarian, or information-seeking MM. Previous studies on MM, though, have neglected the different motivations for second screening. These motivations might impact the way people divide their attention between a television show and a second screen and the manner in which they interact with the two screens, and they might ultimately affect the viewing experience and how consumers process the ads delivered to them. Despite the distinctions that might exist between different kinds of MM, scholars have not examined (a) engagement in two different modes of MM or (b) how these two different modes affect ad processing.

To increase understanding of this issue, the current study introduces two distinctive types of MM: utilitarian MM and hedonic MM, and attempts to examine the situational (i.e., when) predictors of two mode of MM (i.e., hedonic MM and utilitarian MM). In particular, this study proposes program genre as situational factors that affect the types of secondary tasks people engage in while attending to primary media contents. Second, this study aims to investigate how the different modes of MM influences the amount of attention audiences pay to ads appeared on the primary screen and their memory of ads.

\section{Literature review}

\subsection{The Prevalence of Media Multitasking}

MM can be generally defined as "performing two or more tasks simultaneously, one of which involves media use" [8, p. 100]. However, two different views of multitasking have emerged: sequential and concurrent. The sequential multitasking perspective assumes that people cannot encode and process more than one stimulus at a time. From this point of view, multiple tasks are sequentially performed, one at a time, through rapid attention switching between multiple stimuli [9]. Other scholars see multitasking as the result of divided attention, assuming that people can process multiple stimuli concurrently instead of switching between tasks [10]. Considering these two distinct definitions, the current study adopted the sequential multitasking perspective, defining $\mathrm{MM}$ as "sequential engagement in multiple media activities through rapid attention switching."

MM can be categorized into three types: (a) multitasking between a medium and a nonmedia activity (e.g., television and conversation), (b) multitasking between two or more media (e.g., mobile device and television), and (c) multitasking between two different tasks within a single medium (e.g., two tasks opened on a computer. The concept of engaging in other activities while consuming media (e.g., watching TV) is not new. Through in-home observation, it was found that audiences engaged in many complementary (e.g., eating, drinking, conversation about the program) and competitive (e.g., chores and hobbies) activities while watching TV [11]. Although most of the multitasking behavior at this time 
was non-media activity [12], the number of people who concurrently consume multiple media (e.g., the second and third types of MM) is on the rise $[2,13]$. This "new normal" media consumption pattern has emerged primarily because digitalization and increased portability of media devices have allowed people to possess a variety of screens and because the widespread availability of high-speed information has fueled the expectation that human cognitive systems can simultaneously process and respond to multiple sources of information.

While overall media use among U.S adults has increased by $20 \%$ over the past decade, the amount of time people spend $\mathrm{MM}$ has increased more than $119 \%$ over the same period [14]. A recent study conducted by IAB indicated that about $80 \%$ of U.S. adults used another device (e.g., smartphone, laptop, tablet) while watching TV, indicating that MM has become the default mode for media consumers. Another recent study found that combining multiple new media (i.e., simultaneous use of laptop and mobile phone) was more common than combining two traditional media (i.e., simultaneous use of television and magazines) or one traditional medium and one new medium (i.e., simultaneous use of television and a mobile phone) [13].

\subsection{The Effect of MM on Ad Processing}

Previous studies on MM have consistently found the detrimental effect of MM on message processing (i.e., decreased attention, low message exposure, and low memory) based on limited cognitive capacity framework $[4,6]$.

Limited cognitive capacity theory suggests three different levels of information processing: (a) encoding, (b) storage, and (c) retrieval. In the mass communication field, many scholars have operationally equated the "encoding" process with exposure. However, to successfully encode a mediated message, people need to have their sensory receptors engaged (e.g., eyes, ears, skin; Eysenck, 1993) and select which bits of information held in sensory storage will be transformed into mental representations in working memory. That is, without sufficient cognitive resources, such encoding of information cannot occur, suggesting that mere exposure is different from "attention" or "encoding." Because people have a limited pool of attention and mental resources available for cognitive processing [15], MM inevitably interrupts people to encode information. For instance, if an individual searches for an airline ticket on a mobile device during a commercial break for Saturday Night Live, he either divides his attention or rapidly switches his attention between the airline site and the ads on the TV screen $[6,10]$.

This competition for limited cognitive resources is likely to lead to diminished attention to an ad, encoding of sensory input, and ad message recall $[5,10]$. In line with this concept, it was found that people who watched TV programs while reading an article showed significantly lower levels of recall and recognition of the article than those who did not engage in multitasking [4]. The effect of MM has also been found to be detrimental to advertising effectiveness, particularly ad memory [1, 16 -18]. Based on these findings, deficits in ad attention and ad memory are likely to occur when program viewing is combined with other media activities.

H1: Ad attention will be lower when people watch a program while MM (vs. engagement with a single media device).

H2: Brand memory will be lower when people watch a program while MM (vs. engagement with a single media device). 


\subsection{Two Modes of Media Multitasking: Utilitarian vs. Hedonic}

Although MM has often been considered a homogenous phenomenon, the combination of tasks or media can vary, and the effects of different forms of MM might differ [13, 19]. According to a recent report from IAB (2015), the top multi-screening activities in which people engage while watching TV include browsing the Internet, checking email, paying bills, catching up on news, social networking, watching another fun video clips, gaming, and shopping. Among those top multi-screening activities, the first four (i.e., checking email, browsing the Internet, paying bills, and catching up on news) might be defined as more goaldirected, instrumental, and utilitarian media consumption, tasks that people are more strongly motivated to accomplish. On the other hand, the last four (i.e., social networking, watching another fun video clips, gaming, and shopping) might be categorized as more non-directed, navigational, and hedonic media consumption, tasks that are primarily for enjoyment. Paying attention to these differences, the current study introduces two different modes of MM, utilitarian MM and hedonic MM.

In the context of MM, one study identified two motivations for second screening: information seeking and discussion [20]. Also, although the study was limited to online media consumption, media consumption behavior cab be classified into two distinct types: goaldirected and non-goal directed [21]. According to them, the different types of media consumption affect media users' motivation, information processing, and decision-making. In the case of a utilitarian/goal-directed second-screen activity, such as booking a flight ticket or paying a bill, a stronger motivational activation for the task might exist, requiring people to invest a significant amount of their mental resources [22]. On the other hand, in case of a hedonic MM, such as scrolling through posts on Facebook, which usually satisfies media users' hedonic desire or maximizes the enjoyment of media consumption, the level of cognitive effort required is relatively low. Despite such differences between utilitarian MM and hedonic MM, the nature of MM has not been taken into consideration in previous studies. Thus, we examine how media context (i.e., different program genres) affects the occurrence of utilitarian and hedonic MM and how these distinct modes of MM influence ad processing.

\subsection{The Effect of Program Genre on Two Different Modes of MM}

To uncover when people shift their attention from a primary screen to media-related or nonmedia related secondary activities, numerous scholars have explored how media content affects audience attention [23-25). Findings from one observational study indicate that entertaining programs (e.g., comedy) more easily distract audience attention than informational programs (e.g., documentary) [25]. However, findings from a field study conducted by Neilson (2015) indicated the opposite pattern. Although results have been controversial, the effect of program genre on the frequency of MM has received scholarly attention. However, the effect of program genre on the pattern of MM in which people engage remains unexplored.

People tend to save cognitive capacity by avoiding instances involving a high level of cognitive demands [26]. Supporting this perspective, Wang et al. (2015) argued that some MM behaviors can be more cognitively taxing than others and found that people tend to choose MM combinations that are less cognitively demanding in mediated environment [19]. For instance, people tend to choose a secondary task that has a high level of relevance with a primary task, or a MM behavior that has a low level of shared sensory modalities with a primary task (e.g., listening to radio + Facebook) to reduce resource demand. Such findings suggest that the types of MM in which people engage could be influenced by the types of programs being viewed (i.e., primary task). That is, a program that demands high mental resources might increase the extent of hedonic MM, while a program that requires low mental 
load might enable people to engage in both hedonic and utilitarian MM due to surplus of cognitive resources.

In general, programs that fit into the suspense, drama, and thriller genres are cognitively more engaging due to their narrative structure and identification with characters [25]. On the other hand, programs such as sitcoms and reality shows requires less cognitive demand due to the more compartmentalized structure of the show [25]. Since people tend to choose MM behaviors that are less likely to tax their cognitive resources, programs such as suspenseful shows or drama, might lead to fewer cognitively demanding off-task MM behaviors, such as utilitarian MM (e.g., booking a flight ticket, catching up on news). On the other hand, both hedonic MM (e.g., texting to friends) and utilitarian MM are likely to be selected during programs that are cognitively less engaging, such as comedy shows, because people are likely to have enough cognitive resources leftover for secondary tasks. Therefore, the following research question and hypotheses were proposed.

H3: When watching a suspenseful drama, compared to a sitcom, people will engage in a lower level of utilitarian MM during the show (H3a) and commercial break (H3b).

H4: When watching a suspenseful drama or a sitcom, people will engage in the same level of hedonic MM during the show (H4a) and commercial break (H4b).

Furthermore, building on the limited cognitive capacity framework, we predict that people will be more likely to engage in hedonic MM rather than utilitarian MM, regardless of program genre, because people generally choose the combination of tasks that take up a less amount of their cognitive resources to avoid cognitive fatigue [19]. Thus, the following hypothesis is proposed:

H5: In the context of MM, people are more likely to engage in hedonic MM than utilitarian MM regardless of program genre.

\subsection{The Effect of Different Modes of MM on Ad Processing}

According to reversal theory [27], the motivational state of an individual can be one of two types: telic (serious-minded) or paratelic (playful-minded). People in the telic state are likely to be motivated by a goal while people in the paratelic state tend to seek enjoyment and have low goal orientation. Consistent with this concept and the classification of media consumption behavior by Hoffman and Novak (1996) [21], utilitarian MM (e.g., information search, paying a bill) is likely to be more goal-directed and demand a higher level of processing, while hedonic MM (e.g., texting, SNS usage) imposes a relatively small cognitive load.

Because utilitarian MM is likely to be more cognitively taxing than hedonic MM, in turn influencing information loss and communication outcomes $[19,28]$, we predicted that the content people watched on their primary screen would determine their cognitive ad processing. In particular, we predict that people who watch a sitcom might have difficulty processing commercials because the types of the MM in which they engage (i.e., both utilitarian and hedonic) impose a higher cognitive cost. On the other hand, during a suspenseful drama, the detrimental effect of MM on cognitive ad evaluation should lessen because viewers are more likely to engage in hedonic MM than utilitarian MM due to lower available cognitive resources. Thus, we proposed the following hypothesis:

H6: When watching a suspenseful drama, compared to a sitcom, people will show a higher level of ad memory. 


\section{Methodology}

\subsection{Overview of the Research Design}

A lab-based experiment was conducted to examine 1) how program genre affects modes of $\mathrm{MM}$, and b) how different modes of MM worsen or attenuate the detrimental effect of MM. Dependent variables were ad attention, the types of MM engaged and ad memory, which we measured using self-report. The experiment used a 2 (Tasking Type: Single tasking vs. Multitasking) $\times 2$ (Program genre: Suspenseful drama vs. Sitcom) between-subject experimental design.

\subsection{Pretest and Development of Stimulus Material}

Prior to a main experiment, two pretest were conducted (a) to classify different types of MM activities into two categories (hedonic MM vs. utilitarian $\mathrm{MM}$ ), and (b) to choose commercials embedded in the middle of selected programs. In the first pretest, a total of 108 college students participated in the first pretest for extra course credit $\left(M_{\text {age }}=20.90\right.$ years $(S D$ $=1.03), 79.6 \%$ female). To categorize different types of MM activities into two categories (i.e., hedonic MM vs. utilitarian MM), participants were asked to indicate their primary motivations for the top eleven multi-screening activities reported by IAB (2015) on a 7-point scale (1: information seeking, 7: entertainment seeking). The one-sample $t$-test, with a test value of 4 , indicates that information seeking activities included checking email, catching up on the news, paying bills searching information about a show and entertainment seeking activities included texting, browsing the Internet, social networking, watching other fun video clips, gaming, online shopping, tweeting/posting about the show on social networks (See Table 1). We used this classification in the analysis stage to measure the amount of hedonic and utilitarian MM.

Table 1. Classification of Media Multitasking Activities

\begin{tabular}{|l|l|l|l|l|}
\hline Types of Media Multitasking & Mean & SD & $t$ & $p$ \\
\hline Checking Emails & 2.28 & 1.60 & -11.19 & $<.05$ \\
\hline Catching up on News & 2.88 & 1.45 & -8.02 & $<.05$ \\
\hline Paying Bills & 1.87 & 1.18 & -18.68 & $<.05$ \\
\hline Searching Information about A Show & 3.29 & 1.74 & -4.20 & $<.05$ \\
\hline Texting & 5.36 & 1.31 & 10.76 & $<.05$ \\
\hline Browsing the Internet & 5.04 & 1.45 & 7.50 & $<.05$ \\
\hline Social Networking & 5.92 & 1.22 & 16.38 & $<.05$ \\
\hline Watching Other Fun Video Clips & 5.81 & 1.46 & 12.89 & $<.05$ \\
\hline Gaming & 5.27 & 1.89 & 6.70 & $<.05$ \\
\hline Online Shopping & 5.09 & 1.69 & 6.70 & $<.05$ \\
\hline $\begin{array}{l}\text { Tweeting/Posting About The Show On } \\
\text { Social Networks }\end{array}$ & 4.71 & 1.46 & 5.08 & $<.05$ \\
\hline
\end{tabular}

Note: Motivations of Media Multitasking Activities were measured on a 7-point scale (1: Information Seeking, 7: Entertainment seeking).

Source: Own processing.

Next, the second pretest was conducted with a total of with 115 college students $\left(M_{\text {age }}=\right.$ 23.50 years $(S D=1.12), 60.6 \%$ female $)$. To select commercials to embed in the commercial break, we considered a total of fifteen ads across eight different product categories. To eliminate any confounding effects, commercials for unfamiliar brands and commercials that 
were consistent in overall ad characteristics (e.g., attractiveness, credilbity, overall quality of the ad) were selected. Based on these criteria, commercials for Dilmah $\left(\mathrm{M}_{\text {familiarity }}=1.48\right.$, $\mathrm{SD}=1.00)$, NOCS $(\mathrm{M}$ familiarity $=2.32, \mathrm{SD}=2.06)$, Jacobs $(\mathrm{M}$ familiarity $=2.55, \mathrm{SD}=2.21)$, and GoMacro $\left(\mathrm{M}_{\text {familiarity }}=1.83, \mathrm{SD}=1.50\right.$ were chosen.

Based on the pretests, we developed stimulus materials. For each 10-minute clip of the suspenseful dramas (Blacklist and Luther) and sitcoms (Hot in Cleveland and 2 Broke Girls), we embedded the four selected ads as a commercial break.

\subsection{Manipulation}

Tasking Type. To manipulate tasking type, we assigned participants one of two tasking conditions: (a) watch a TV program without access to another media device and (b) watch a TV program and freely use a mobile phone (except to make phone calls).

Program Genre. To manipulate program genre, those assigned to the suspenseful drama condition watched Blacklist or Luther while those assigned to the sitcom condition watched Hot in Cleveland or 2 Broke Girls.

\subsubsection{Dependent Measures}

We measured a total of three dependent variables to assess the effect of program genre on MM and the effect of MM on ad evaluations: (a) amount of attention paid to commercial, (b) types of MM, and (c) ad memory. Ad attention was measured using two seven-point Likert items adopted from Chaffee and Schleuder (1986) [29]. The items included "How much attention did you pay to the commercials while watching the video clip?" and "How much did you focus on the commercials embedded in the video clip?" The types of MM activities in which the participants engaged were measured by asking them to list all of the mobile phone tasks they performed while watching the program and commercials, respectively. To increase the accuracy of their memory, a list of eleven prominent MM activities reported by IAB were given as examples, and they were allowed to look through the browser history on their mobile phones. Then, based on the classification of two types of MM (see Table 1), we generated a composite score for hedonic MM and utilitarian MM, respectively, by adding the number of activities participants reported. Lastly, we measured ad memory using two indicators: ad recall and ad recognition. For ad recall, participants wrote down any brands they could remember seeing during the commercial break [1]. For ad recognition, we provided a list of ten brands (i.e., four target brands and six filler brands); participants then chose as many brands as they could remember seeing during the commercial break [18]. We derived the ad recognition score by subtracting the number of incorrectly chosen brands from the number of correctly chosen brands.

\subsubsection{Participants and Procedure}

A total of 165 undergraduate courses enrolled at a large southeastern public university in the United States took part in the experiment $(82.4 \%$ female; 19 to 30 years old $[M=21.54, S D$ $=2.20]$ ). When the participants signed up, they were asked to bring their mobile phone the day of the experiment. Each student participated in a 60-minute lab session, which took place in a research lab equipped with a computer station. When participants arrived in the lab, they were seated in front of a 27-inch iMac computer and asked to choose one of the four programs available. Then, we randomly assigned participants to either the single-tasking or multitasking condition. To help participants naturally engage in MM behavior, the researcher left the lab once the participants began watching the selected program on their computer 
screen. Among those in MM condition, $91.4 \%$ of them reported that they used an Apple iPhone $(5,5 \mathrm{~s}, 6,6+$, or 7); the rest reported using a Samsung Galaxy (6 or S7).

\section{Results}

First, to test the effect of media multitasking on ad attention and ad memory, we conducted independent sample $t$-tests. Confirming previous findings and our predictions, people who engaged in MM not only paid less attention to the program but also showed a lower level of ad memory compared to those who watched a TV program without access to a secondary device (See Table 2). Thus, H1 was supported, while H2 was partially supported.

Table 2. Media Multitasking Effect

\begin{tabular}{|l|c|c|c|c|}
\hline & Single tasking & Multitasking & & \\
\hline Ad attention & Mean (SD) & Mean (SD) & $t$ & $p$ \\
\hline Ad recall & $4.59(1.39)$ & $2.82(.68)$ & 6.33 & $<.001$ \\
\hline Ad recognition & $.53(.90)$ & $.82(.07)$ & 1.58 & .12 \\
\hline
\end{tabular}

Source: Own processing.

Next, to test whether different program genre influenced the type of MM activities in which participants engaged, independent sample $t$-tests were conducted. This study posited that the degree to which people engage in utilitarian MM would vary with program-genre, for the amount of cognitive resources leftover for secondary activities differ. As expected, participants who watched a sitcom (i.e., Hot in Cleveland or 2 Broke Girls) tended to engage in a higher level of utilitarian MM (e.g., checking email, catching up on the news) than those who watched a suspenseful drama (i.e., The Blacklist or Luther) not only during the program $\left(M_{\text {drama }}=.38, S D=.59\right.$ vs. $\left.M_{\text {sitcom }}=66, S D=.75, t=-2.23, p<.01\right)$ but also during the commercial break $\left(M_{\text {drama }}=.39, S D=.53\right.$ vs. $\left.M_{\text {sitcom }}=.79, S D=.78, t=-3.19, p<.01\right)$. However, the amount of hedonic MM was not significantly different between the two programs (during the program: $M_{\text {drama }}=1.28, S D=.87$ vs. $M_{\text {sitcom }}=1.59, S D=.90, t=-1.93$, $p>.05$; during the commercial break: $M_{\text {drama }}=1.83, S D=.98$ vs. $M_{\text {sitcom }}=1.90, S D=.91, t$ $=-.43, p>.05$ ). Thus, $\mathrm{H} 3$ and $\mathrm{H} 4$ were supported (Fig. 1.).
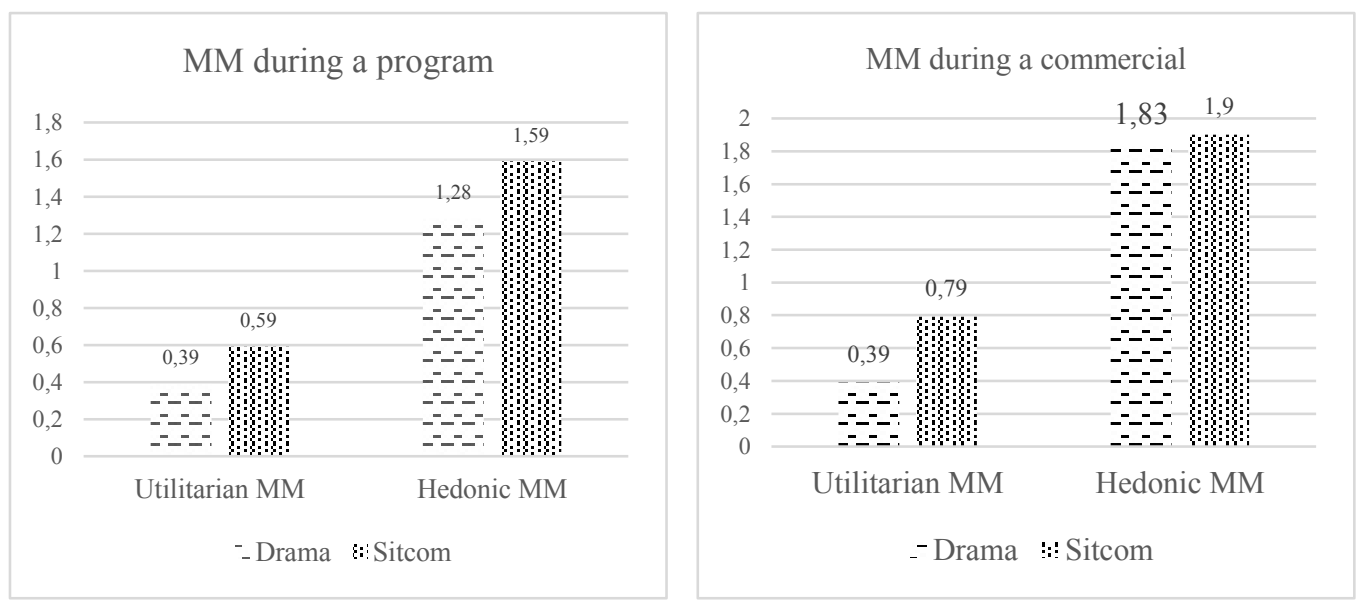

Fig. 1. The Effect of Program Genre on Modes of Media Multitasking Source: Own processing. 
To test H5, which predicted the relative amount of hedonic vs. utilitarian MM for each program genre, we conducted paired sample $t$-tests. Findings suggest that people were more likely to engage in hedonic or entertaining MM (e.g., texting, SNS, gaming) than utilitarian or information-seeking MM (e.g., catching up on the news, searching information) both when watching a suspenseful drama (during program: $M_{\text {hedonic }}=1.28, S D=.87$ vs. $M_{\text {utilitarian }}=.38$, $S D=59, t=12.97, p<.001$; during commercial break: $M_{\text {hedonic }}=1.83, S D=.98$ vs. $M_{\text {utilitarian }}$ $=.40, S D=53, t=8.22, p<.001$ ) and when watching a sitcom (during program: $M_{\text {hedonic }}=$ $1.59, S D=.90$ vs. $M_{\text {utilitarian }}=.66, S D=.75, t=7.86, p<.001$; during commercial break: $M_{\text {hedonic }}=1.90, S D=.91$ vs. $\left.M_{\text {utilitarian }}=.79, S D=.78, t=7.63, p<.001\right)$. Thus, H5 was supported.

Lastly, in MM context, we predicted that people who watched a drama would show a higher level of ad memory as they mainly engage in hedonic MM, while those who watch a sitcom engage in both hedonic MM and utilitarian MM at a greater extent, which is likely to diminish one's cognitive capacity. To test the hypothesis, independent t-tests were performed. Among those who freely used their smartphones while watching a TV program on a computer screen, those who watched a suspenseful drama showed a significantly higher level of ad recall $\left(M_{\text {drama }}=.48, S D=.86\right.$ vs. $\left.M_{\text {sitcom }}=.13, \mathrm{SD}=.74, \mathrm{t}=2.40 p<.05\right)$ and ad recognition $\left(M_{\text {drama }}=2.04, S D=1.25\right.$ vs. $\left.M_{\text {sitcom }}=1.40, S D=1.07, \mathrm{t}=2.85 p<.01\right)$, supporting H6. It should be also noted that these differences in ad memory was not found in single-tasking group (Table 3).

Table 3. The Moderating Effect of Program Genre on Ad Memory in the Context of MM

\begin{tabular}{|l|c|c|c|c|}
\hline & Drama & Sitcom & $t$ & $p$ \\
\cline { 2 - 5 } & Mean (SD) & Mean (SD) & & \\
\hline Ad recall & $.48(.86)$ & $.13(.74)$ & 2.40 & $<.05$ \\
\hline Ad recognition & $2.04(1.25)$ & $1.40(1.07)$ & 2.85 & $<.01$ \\
\hline
\end{tabular}

Source: Own processing.

\section{Discussion}

The purpose of this study was to understand a) distinct types of MM, b) the effect of programgenre on the occurrence of different types of MM, and c) its subsequent effect on ad processing. Supporting previous findings [4-6], the current study confirmed the negative effect of MM on attention and memory. When the computer and mobile device were simultaneously accessible, participants generally showed a lower level of ad attention and ad recognition than participants in the single-task group (i.e., computer only). The current study also found that the content people watch on their primary screen determines the modes of MM in which they engage, in turn influencing their cognitive ability to process brand-related information. Supporting the limited cognitive capacity framework [15], our findings indicate that those who watched a cognitively demanding suspenseful drama tended to engage in a lower amount of utilitarian MM than those who watched a sitcom, while both groups engaged in a similar amount of hedonic MM. According to Wang et al. (2015) [15], the cognitive demand of MM depends on task combination, in turn influencing a media users' ability to process information on a primary and secondary screen. Wise et al. (2009) found that a utilitarian or goal-oriented task (e.g., searching information) activated strong motivation than a hedonic or low-purpose media task (e.g., texting friends) [22]. In line with this idea, we found that participants who watched a suspenseful drama showed a higher level of ad recall and ad recognition than those who watched a sitcom, for the former tended to engage in a lower amount of utilitarian MM, which is cognitively more taxing. 
The contribution of this study is twofold. First, the previous studies, which examined the media multitasking phenomenon, have neglected audiences' different motivations for second screening activities. To fill this gap, this study introduced two distinctive nature of MM: utilitarian $M M v s$. hedonic $M M$, and identified the predictors and consequences of different modes of MM. Second, we increased the validity of our findings by creating a more natural media consumption environment. Although previous studies have examined MM in many different forms (e.g., TV screen + Tablet: [18]; Multitasking on a single computer: [1,17]), few studies have investigated this issue in light of the most predominant pattern of MM among college students. To fill this gap, the current study investigated the simultaneous use of computers and mobile devices in a realistic setting.

Although the current study has meaningful implications, the results should be carefully interpreted due to several limitations. First, although we increased the ecological validity of the findings by allowing participants to engage in MM using their own smartphones, this approach might have created confounding effects. For instance, we have found that participants engaged in different amounts and types of secondary activities, potentially affecting their ad processing to varying degrees. Second, we assumed that those who watched a suspenseful drama showed a higher level of ad memory than those who watched a sitcom due to different amounts of utilitarian MM in which they engaged; however, we did not directly test this underlying mechanism in the current study. Thus, based on our findings, future researchers should consider investigating the effect of different types of MM on ad processing in a more controlled setting.

Lastly, the findings are limited to college students aged 20-30 years due to the samples used in the study. Although computers and mobile devices were reported as the most predominant $\mathrm{MM}$ combination among college students, other age groups might exhibit different patterns of MM (e.g., TV + mobile device). Although the student sample used in the current study is justifiable because this age group tends to engage in MM more than other age groups, future studies might consider the behavior of other age groups that constitute important targets of advertising. In addition, although individual differences were not considered in the current study, personal characteristics (e.g., attachment style or need for closure) might affect the type of MM behavior in which individuals engage.

\section{Conclusion}

In conclusion, although many scholars have considered MM a homogenous phenomenon, our experimental findings suggest that $\mathrm{MM}$ can be classified into two distinct modes: utilitarian $M M$ and hedonic MM. Further, it offers the empirical evidence that modes of MM consumers engage in determine consumers' cognitive ability to process brand-related information on the primary screen. Similar to previously identified factors that moderated the detrimental effect of MM on ad processing and memory (e.g., ad appeal [17], processing style [1], congruence between primary and secondary screen activity [30]), this study suggests that program genre determines the types of MM consumers engage in, and eventually have the power to attenuate or worsen the negative effect of $\mathrm{MM}$ on ad memory. We hope these findings provide useful guideline on media planning for advertisers, and help motivate future researchers to build on the current research and continue to deepen our understanding in the area of MM. 


\section{References}

1. B. R. L. Duff, S. Sar, Seeing the big picture: Multitasking and perceptual processing influences on ad recognition. Journal of Advertising, 44(3), 173-184 (2015)

2. E. Ophir, C. Nass, A. D. Wagner, Cognitive control in media multitaskers. Proceedings of the National Academy of Sciences, 106(37), 15583-15587 (2009)

3. Z. Wang, J. M. Tchernev, The "myth" of media multitasking: Reciprocal dynamics of media multitasking, personal needs, and gratifications. Journal of Communication, 62(3), 493-513 (2012)

4. G. B. Armstrong, L. Chung, Background television and reading memory in context: Assessing TV interference and facilitative context effects on encoding versus retrieval processes. Communication Research, 27(3), 327-352 (2000)

5. M. L. Courage, A. Bakhtiar, C. Fitzpatrick, S. Kenny, K. Brandeau, Growing up multitasking: The costs and benefits for cognitive development. Developmental Review, 35, 5-41 (2015)

6. W. Zhang, S. H. Jeong, M. Fishbein, Effects of multitasking and arousal on television content recall and secondary task performance. Journal of Media Psychology, 22(1), 2$13(2010)$

7. S. Chinchanachokchai, B. R. Duff, S. Sar, The effect of multitasking on time perception, enjoyment, and ad evaluation. Computers in Human Behavior, 45, 185-191 (2015)

8. A. Lang, J. Chrzan, Media multitasking: Good, bad, or ugly? Annals of the International Communication Association, 39(1), 99-128 (2015)

9. E. Wood, L. Zivcakova, P. Gentile, K. Archer, D. De Pasquale, A. Nosko, Examining the impact of off-task multi-tasking with technology on real-time classroom learning. Computers \& Education, 58(1), 365-374 (2012)

10. B. R. Duff, C. M. Segijn, Advertising in a media multitasking era: Considerations and future directions. Journal of Advertising, 48(1), 27-37 (2019)

11. D. M. Krugman, G. T. Cameron, C. M. White, Visual attention to programming and commercials: The use of in-home observations. Journal of Advertising, 24(1), 1-12 (1995)

12. S. H. Jeong, M. Fishbein, Predictors of multitasking with media: Media factors and audience factors. Media Psychology, 10(3), 364-384 (2007)

13. H. A. Voorveld, C. M. Segijn, P. E. Ketelaar, E. G. Smit, Investigating the prevalence and predictors of media multitasking across countries. International Journal of Communication, 8, 2755-2777 (2014)

14. M. W. Becker, R. Alzahabi, C. J. Hopwood, Media multitasking is associated with symptoms of depression and social anxiety. Cyberpsychology, Behavior, and Social Networking, 16(2), 132-135 (2013)

15. A. Lang, The limited capacity model of mediated message processing. Journal of communication, 50(1), 46-70 (2000)

16. P. D. Bolls, D. D. Muehling, The effects of dual-task processing on consumers' responses to high-and low-imagery radio advertisements. Journal of Advertising, 36(4), 35-47 (2007)

17. S. Kazakova, V. Cauberghe, L. Hudders, C. Labyt, The impact of media multitasking on the cognitive and attitudinal responses to television commercials: The moderating role of type of advertising appeal. Journal of Advertising, 45(4), 403-416 (2016) 
18. C. M. Segijn, H. A. Voorveld, E. G. Smit, The underlying mechanisms of multiscreening effects. Journal of Advertising, 45(4), 391-402 (2016)

19. Z. Wang, M. Irwin, C. Cooper, J. Srivastava, Multidimensions of media multitasking and adaptive media selection. Human Communication Research, 41(1), 102-127 (2015)

20. H. Gil de Zúñiga, V. Garcia-Perdomo, S. C. McGregor, What is second screening? Exploring motivations of second screen use and its effect on online political participation. Journal of communication, 65(5), 793-815 (2015)

21. D. L. Hoffman, T. P. Novak, Marketing in hypermedia computer-mediated environments: Conceptual foundations. Journal of marketing, 60(3), 50-68 (1996)

22. K. Wise, H. J. Kim, J. Kim, The effect of searching versus surfing on cognitive and emotional responses to online news. Journal of Media Psychology, 21(2), 49-59 (2009)

23. C. G. Christensen, D. Bickham, C. S. Ross, M. Rich, Multitasking with television among adolescents. Journal of broadcasting \& electronic media, 59(1), 130-148 (2015)

24. R. P. Hawkins, S. Pingree, J. Hitchon, B. Radler, B. W. Gorham, L. Kahlor, G. H. Kolbeins, What produces television attention and attention style? Genre, situation, and individual differences as predictors. Human communication research, 31(1), 162-187 (2005)

25. H. A. Voorveld, V. Viswanathan, An observational study on how situational factors influence media multitasking with TV: The role of genres, dayparts, and social viewing. Media Psychology, 18(4), 499-526 (2015)

26. W. Kool, J. T. McGuire, Z. B. Rosen, M. M. Botvinick, Decision making and the avoidance of cognitive demand. Journal of Experimental Psychology: General, 139(4), 665 (2010)

27. M. J. Apter, Reversal theory and personality: A review. Journal of Research in Personality, 18(3), 265-288 (1984)

28. H. Bang, K. King, The effect of media multitasking on ad memory: the moderating role of program-induced engagement and brand familiarity. International Journal of Advertising, 1-30 (2020)

29. S. H. Chaffee, J. Schleuder, Measurement and effects of attention to media news. Human communication research, 13(1), 76-107 (1986)

30. R. Angell, M. Gorton, J. Sauer, P. Bottomley, J. White, Don't distract me when I'm media multitasking: Toward a theory for raising advertising recall and recognition. Journal of Advertising, 45(2), 198-210 (2016) 